\title{
O Índice Laterítico no mapeamento do regolito: Porção Nordeste do Cráton São Francisco, Brasil
}

Rodrigo Soares Vieira dos Santos ${ }^{1}$, Edgar Romeo Herrera de Figueiredo Iza ${ }^{1}$ e Basílio Elesbão da Cruz Filho'

\author{
${ }^{1}$ CPRM - Companhia de Pesquisa de Recursos Minerais \\ E-mail: rodrigo.vieira@cprm.gov.br \\ Copyright 2019, SBGf - Sociedade Brasileira de Geofísica \\ This paper was prepared for presentation during the $16^{\text {th }}$ International Congress of the \\ Brazilian Geophysical Society held in Rio de Janeiro, Brazil, 19-22 August 2019. \\ Contents of this paper were reviewed by the Technical Committee of the $16^{\text {th }}$ \\ International Congress of the Brazilian Geophysical Society and do not necessarily \\ represent any position of the SBGf, its officers or members. Electronic reproduction or \\ storage of any part of this paper for commercial purposes without the written consent \\ of the Brazilian Geophysical Society is prohibited.
}

\begin{abstract}
The regolith mapping is essential for the understanding of supergenic, pedological and geomorphological processes of a region and strongly contributes to the mineral exploration, mainly of weathering-related metallic minerals. Although used successfully in several countries, with emphasis on Australia, the methodology is still poorly applied in Brazil. The present work aims to verify the efficiency of aerogamaspectrometric data in the regolith characterization of an area in the northeast portion of the São Francisco Craton, a region that englobes well-known supergenic minerals occurrences ( $\mathrm{Al}, \mathrm{Fe}, \mathrm{Ni}, \mathrm{Mn}$, etc.). For this purpose, the lateritic index map (Iza, 2017), which is the product of the $T h / K$ and $U / K$ ratios, is used, highlighting the areas which are rich in Th and $U$ and poor in $\mathrm{K}$, and therefore with a higher probability to contain well-developed lateritic profiles. The data were compared with field data in order to verify if the materials with different degrees of laterization have well-defined gamma spectrometric signatures.
\end{abstract}

\section{Introdução}

O regolito é representado por todo material inconsolidado ou secundariamente recimentado sobreposto ao embasamento inalterado, que tenha sido formado pelo intemperismo, erosão, transporte e deposição a partir de materiais pretéritos (Eggleton, 2001). O regolito inclui, portanto, o embasamento alterado e fraturado, saprolitos, solos, acumulações orgânicas, material vulcânico, depósitos glaciais, colúvio, alúvio, sedimentos evaporíticos, depósitos eólicos e água subterrânea.

O estudo do regolito tem permitido a compreensão dos processos supergênicos, pedológicos, geomorfológicos e contribuído fortemente para prospecção mineral, por ser fonte de minerais metálicos ( $\mathrm{Al}, \mathrm{Fe}, \mathrm{Mn}, \mathrm{Ni}, \mathrm{Cu}, \mathrm{Pb}, \mathrm{Au}$, ETR, etc.), além de agregados para construção civil (Taylor e Eggleton, 2001). A lateritização é um dos importantes processos envolvidos na formação do regolito (solos, crostas lateríticas, aluvião, etc.), no enriquecimento supergênico e no modelamento da paisagem, ocorrendo sob condições de clima tropical (Freyssinet et al. 2005).

$\mathrm{Na}$ Austrália, diversas pesquisas destacam que o mapeamento do regolito integrado ao controle do relevo é importante para a compreensão dos processos supergênicos (Anand e Paine, 2002). Smith et al. (2000), por exemplo, ressaltaram que pelo menos 13 depósitos de ouro foram descobertos na Austrália entre 1983 e 1994, por meio de estudos geoquímicos dos perfis lateríticos integrados ao mapeamento do regolito.

Por outro lado, áreas com presença significativa de perfis lateríticos bem desenvolvidos (com crostas lateríticas) são conhecidas em outras partes do mundo, especialmente associadas ao cinturão intertropical (latitudes menores que $30^{\circ}$ ). No Brasil, o estudo do regolito laterítico com ênfase aos aspectos geológicos, geofísicos e geocronológicos ainda é pouco difundido, apesar dos lateritos serem a fonte principal de Al (bauxita) e do processo laterítico aumentar a concentração de vários elementos como $\mathrm{Sn}, \mathrm{Au}, \mathrm{Mn}, \mathrm{Ni}$, $P$, muitas vezes viabilizando sua explotação econômica (Costa, 2007).

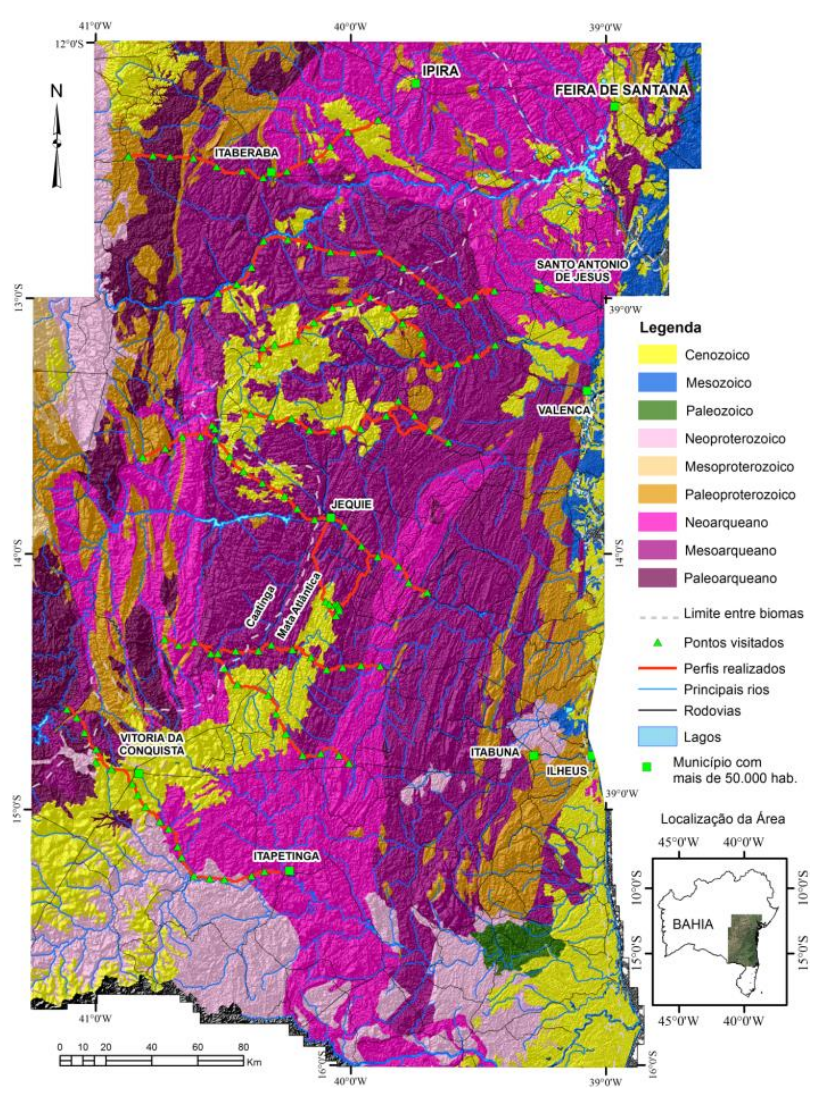


Figura 1 - Mapa geológico e de localização da área estudada. Modificado de Souza et al. (2003).

Apesar da existência de dados aerogeofísicos recentes, não há registro de caracterização e/ou estudo do regolito na porção nordeste do Cráton São Francisco (sudeste da Bahia). Além disso, a ocorrência de diversos recursos minerais com componentes relacionadas aos processos supergênicos, tais como $\mathrm{Al}, \mathrm{Fe}, \mathrm{Ni}$, etc. credencia esta região (Figura 1) como forte candidata a estudos relacionados ao intemperismo e aos próprios processos supergênicos associados.

\section{Metodologia}

A aerogamaespectrometria é utilizada largamente na pesquisa mineral, mapeamento geológico, mapeamento do regolito e das crostas lateríticas devido a sua capacidade de diferenciar assinaturas radiométricas de diferentes litologias. (McQueen e Graig, 1995; Wilford et al., 1997; Minty, 1997; Carrino, et al., 2009 e 2011a; Minty, 2011; Wilford, 2012 e 2013; Arhin et al., 2015, Herrera, 2016a; Iza, 2018, entre outros).

Devido à sua alta solubilidade, a concentração de $K$ geralmente diminui com o aumento do intemperismo, tendendo a ser lixiviado de minerais primários (micas, Kfeldspatos, etc.) ou ser absorvido em argilominerais. Já o $\mathrm{U}$ e o Th são considerados menos móveis que o $\mathrm{K}$. Quando liberado de minerais pelo intemperismo, o $U$ é mais móvel em condições oxidantes, mas precipita em condições redutoras. Embora possa se tornar móvel em combinação com complexos orgânicos em solos e água subterrânea, o Th tende a se concentrar no regolito, por meio de minerais resistatos e óxidos de ferro (Wilford et al, 1997).

No intuito de analisar de forma mais aprofundada a relação entre 0 intemperismo $e$ os dados gamaespectrométricos, foi gerado o índice laterítico (Figura 2) de acordo com o proposto por Iza (2017). O índice laterítico (equação 1) propiciou a avaliação das áreas com diferentes níveis de intemperismo (maior ou menor probabilidades de lateritização). Nele são utilizadas as razões $\mathrm{Th} / \mathrm{K}$ e U/K que resultam de acordo com Iza (2017), na seguinte equação:

$$
\text { Eq.1 IL }=\frac{\text { eTh } x \text { eU }}{\mathrm{K}^{2}}
$$

onde IL é o índice laterítico; eTh é o canal do tório; eU é o canal do urânio e $\mathrm{K}$ refere-se ao canal do potássio.

Foi realizada uma etapa de campo que focou na realização de 8 perfis de direção aproximada leste-oeste com extensão total aproximada de $1700 \mathrm{~km}$. O objetivo desta etapa foi correlacionar os dados de campo com o mapa do índice laterítico. Ao todo foram visitados 118 pontos, onde foram descritos os afloramentos rochosos e o próprio regolito (rochas, solos, crostas lateríticas ferruginosas e aluminosas, horizonte mosqueado, saprolito, etc.).

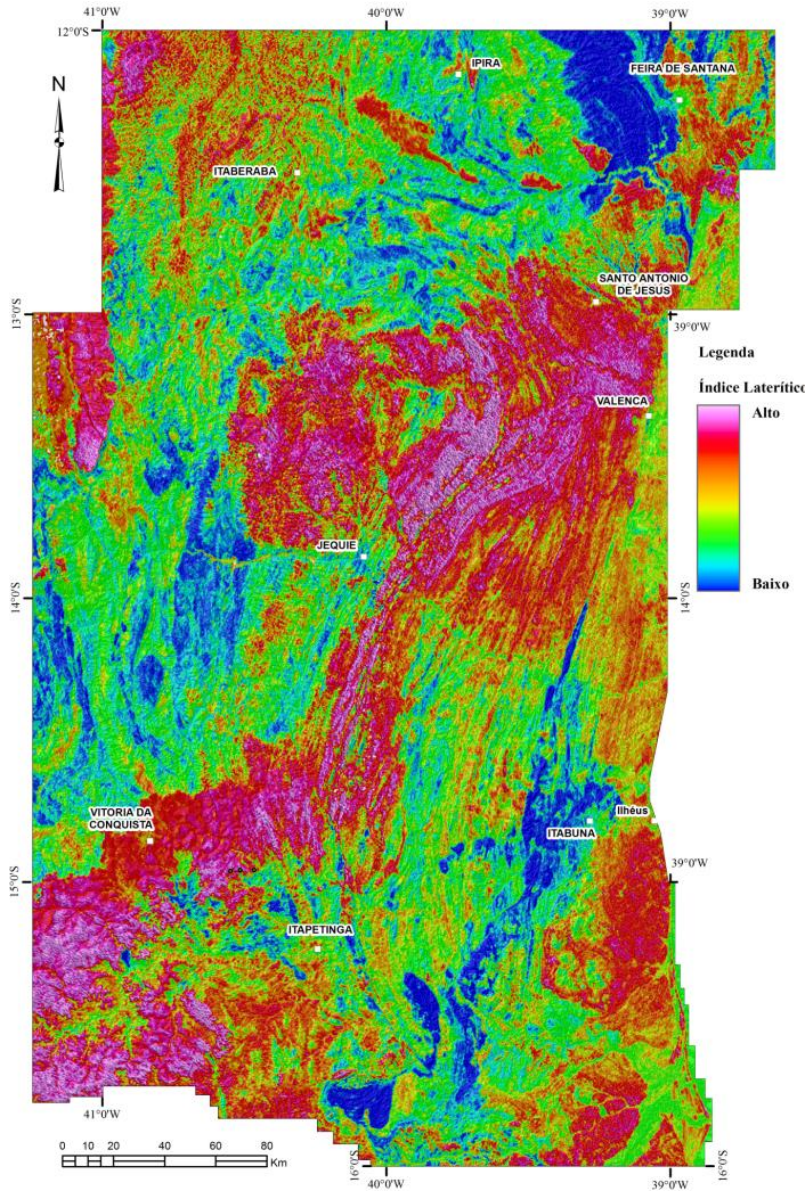

Figura 2 - Mapa do índice laterítico destacando em vermelho e rosa escuro as áreas com alto índice e, portanto, com alto potencial para ocorrência de crostas lateríticas e latossolos associados.

\section{Resultados}

A visita aos 118 pontos propiciou a identificação dos mais variados materiais que constituem o regolito. Nesse aspecto, os dados de campo foram sobrepostos a imagem do IL para posterior correlação entre os materiais (solo, horizonte mosqueado, saprolito, rocha sã, etc.) e seus respectivos valores. O objetivo foi destacar regionalmente os principais domínios de acordo com os materiais predominantes.

A partir desses padrões e da comparação com os dados de campo, foram definidos valores de corte que caracterizam a assinatura gamaespectrométrica de 3 domínios, utilizados na classificação do mapa do índice laterítico (Figura 3). No domínio 1 (preto na Figura 3, valores de IL mais baixos), predominam afloramentos de rocha sã, além de saprolito e solos pouco desenvolvidos. No domínio 2 (verde na Figura 3, valores intermediários de IL), ocorre a alternância entre solos poucos desenvolvidos e rocha alterada. Já no domínio 3 (azul na Figura 3, valores mais altos de IL), ocorre o predomínio de perfil laterítico bem desenvolvido (Figura 4), podendo ocorrer crostas lateríticas ferruginosas e aluminosas. 


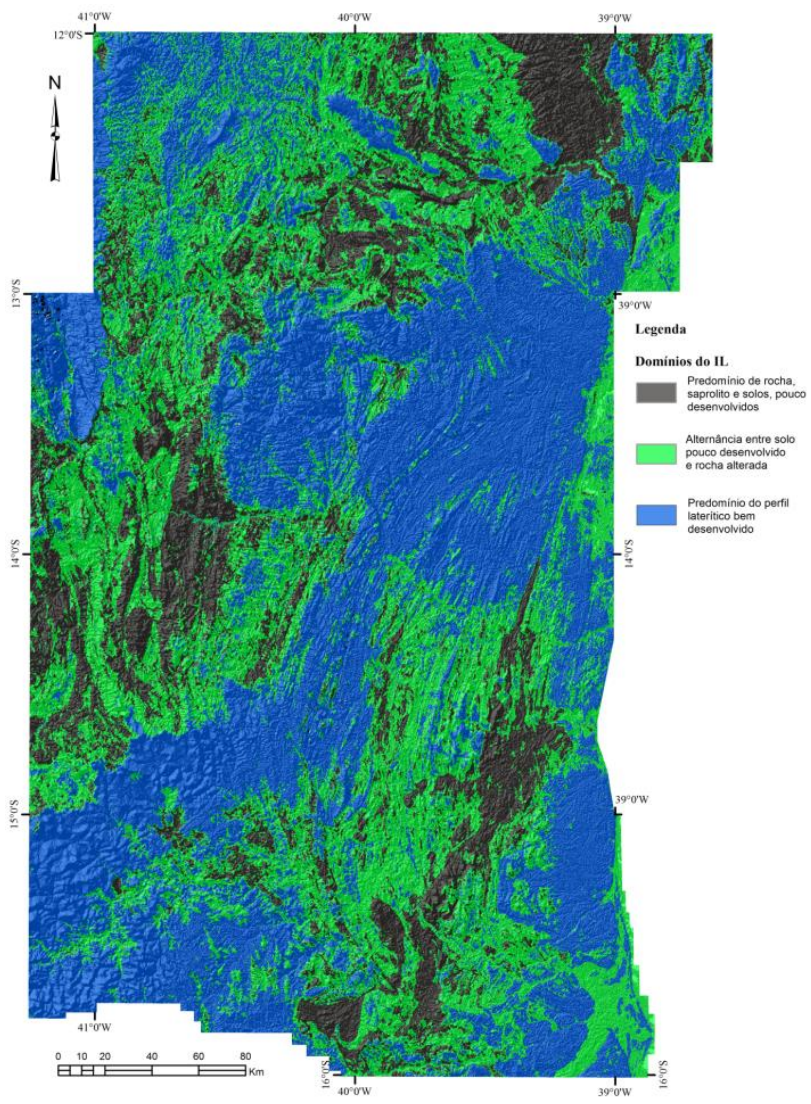

Figura 3 - Mapa de domínios do índice laterítico destacando os três principais domínios individualizados com apoio dos dados de campo.

\section{Conclusões}

A partir da análise dos resultados obtidos e comparação com as áreas que possuem mapeamento geológico, conclui-se que o índice laterítico pode caracterizar de forma satisfatória o perfil intempérico de uma região.

Os domínios observados mostraram ter boa correlação com as unidades geológicas mapeadas, com o domínio 3 normalmente coincidindo com unidades descritas como coberturas detrito-lateríticas e coberturas detrito lateríticas indiferenciadas, ratificando o potencial do produto utilizado.

Visando à exploração de recursos minerais supergênicos, propõe-se o uso do índice laterítico integrado com imagens de sensoriamento remoto, análises geoquímicas, entre outros métodos, para atingir resultados mais robustos.

\section{Agradecimentos}

- CPRM, Companhia de Pesquisa de Recursos Minerais.
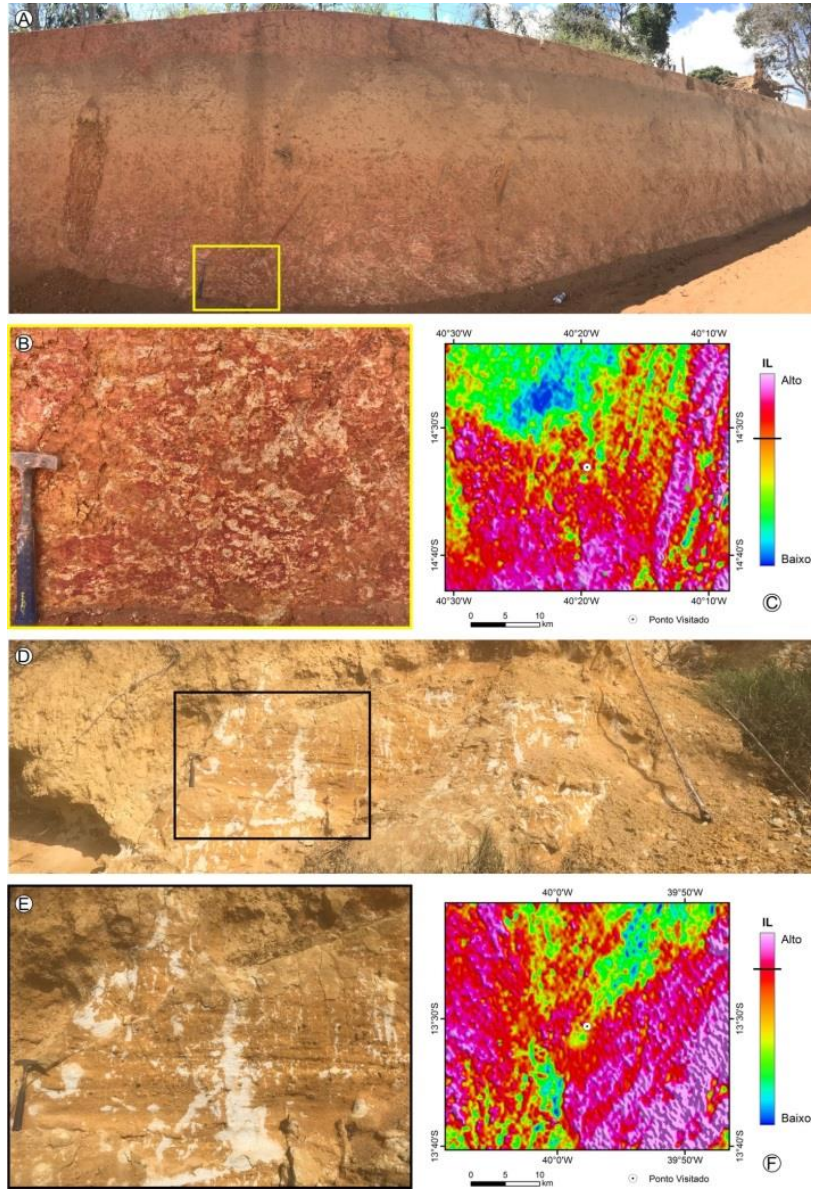

Figura 4 - Exemplo de dominância de solo espesso com a presença de horizonte mosqueado. A) Aspecto geral do solo argiloso de cor avermelhada (topo) e horizonte mosqueado (base). B) Detalhe do horizonte mosqueado C) Contexto gamaespectrométrico (IL). D) Aspecto geral de outro horizonte mosqueado. E) Detalhe do horizonte mosqueado com porções brancas e vermelhas. F) Contexto gamaespectrométrico (IL).

\section{Referências Bibliográficas}

ARHIN, E.; JENKIN, G.R.T.; CUNNINGHAM, D.; NUDE, O. 2015. Regolith mapping of deeply weathered terrain in savannah regions of the Birimian Lawra Greenstone Belt, Ghana. Journal of Geochemical Exploration, 159, 194207.

ANAND, R.R.; PAINE, 2002. M. Regolith geology of Yilgarn Craton, western Australia: Implication for exploration. Australian Journal of Earth Sciences, 49, 3162.

CARRINO, T. A.; SILVA, A.M.; BOTELHO, N.F.; SILVA, A.A.C. 2009. Detecção de ocorrências de coberturas supergênicas a partir de imagens altimétricas e gamaespectrométricas: os alvos do extremo leste do Amazonas Anais XIV Simpósio Brasileiro de 
Sensoriamento Remoto, Natal, Brasil, INPE, p. 31973204.

CARRINO, T. A.; SILVA, A.M.; BOTELHO, N.F.; SILVA, A.A.C. 2011a. Discriminação de áreas de espesso regolito do leste do Estado do Amazonas usando estatística multivariada, algoritmo hiperespectral e modelagem de dados espaciais. Revista Brasileira de Geofísica, v. 29, n. 1, p. 155-172.

COSTA M.L 2007. Introdução Ao Intemperismo Laterítico E À Lateritização In: Prospecção Geoquímica De Depósitos Minerais Metálicos, Não Metálicos, Óleo E Gás. Otavio Licht, Carlos Siqueira Bandeira Mello, Cássio Roberto da Silva.. Rio de Janeiro: Sociedade Brasileira de Geoquímica - SBGq / CPRM - Serviço Geológico do Brasil , p. 199-244. ISBN 9788574990.

EGGLETON R. A. (Editor) 2001. Glossary of RegolithSurficial Geology, Soils and Landscapes. CRC LEME Publication, Perth.

FREYSSINET, Ph., BUTT, C.R.M., MORRIS, R.C. \& PIANTONE, P. 2005, Ore-forming processes related to lateritic weathering. Economic Geology, 100th Aniversary Volume, 681-722.

HERRERA, I.L.I.E. Utilização de dados altimétricos, geomorfológicos e gamaespectrométricos para a identificação de crostas lateríticas em uma área da porção norte do estado de Rondônia. 2016a. Dissertação (Mestrado em Geografia). Universidade Federal de Rondônia. Porto Velho. 2016.

IZA, E.R.H.F. Coberturas lateríticas do SW do Cráton Amazônico: aspectos geofísicos e geoquímicos. 2017. xvi, 73 f., il. Tese (Doutorado em Geologia)Universidade de Brasília, Brasília.

McQUEEN K.G.; CRAIG, M. A. (1995) Developments and New Approaches in Regolith Mapping. Centre for Australian Regolith Studies. Occasional Publication no. 3. Australian National University University of Canberra.

MINTY, B.R.S., 1997. Fundamentals of airborne gammaray spectrometry. AGSO Journal of Australian Geology and Geophysics 17, 39-50.

MINTY, B. (2011). Short note: on the use of radioelement ratios to enhance gamma-ray spectrometric data. 559 Exploration Geophysics. 42(1), 116-120. doi: 10.1071/EG10011

SMITH, R.E; ANAND, A.R.R; ALLEY, N.F 2000. Use and implications of paleoweathering surfaces in mineral exploration in Australia. Ore Geology Reviews 16_2000. 185-204.

TAYLOR, G.; EGGLETON, R. A. 2001. Regolith geology and geomorphology. Chichester: John Wiley.

WILFORD, J. R.; BIERWIRTH, P.N.; CRAIG, M.A. 1997 Application of airborne gamma-ray spectrometry in soil/regolith mapping and applied geomorphology. Journal of Australian Geology \& Geophysics, v. 17, n. 2, p. 201216.

WILFORD, J.R, 2012. A weathering intensity index for the Australian continent using airborne gamma-ray spectrometry and digital terrain analysis. Geoderma 183184, $124-142$. http://doi.org/10.1016/j.geoderma.2010.12.02.

WILFORD, J.R, 2013. New regolith mapping aproaches for old australian landscapes. PhD thesis, The University of Adelaide. South Australia. 